\title{
Optical Frequency Domain Reflectometry: A Review
}

\author{
Kivilcim Yuksel, Marc Wuilpart, Véronique Moeyaert, and Patrice Mégret \\ Faculté Polytechnique de Mons, Boulevard Dolez 31, 7000 Mons, Belgium \\ kivilcim.yuksel@fpms.ac.be
}

\begin{abstract}
In this paper, we discuss the operation principles, challenges and application areas of optical frequency domain reflectometry technique. A review of OFDR systems is given considering both Incoherent-OFDR and CoherentOFDR groups. Results obtained by different research groups are compared in terms of performance characteristics, and future perspectives. Recent progresses obtained within our laboratory are also presented.

Keywords: optical frequency domain reflectometer (OFDR), nonlinear optical frequency sweep
\end{abstract}

\section{OPTICAL FREQUENCY DOMAIN REFLECTOMETRY - OFDR}

OFDR methods fall into two main categories: incoherent OFDR (I-OFDR) and coherent OFDR (C-OFDR). Figure 1 summarizes the different trends in OFDR that have been published in the scientific literature.

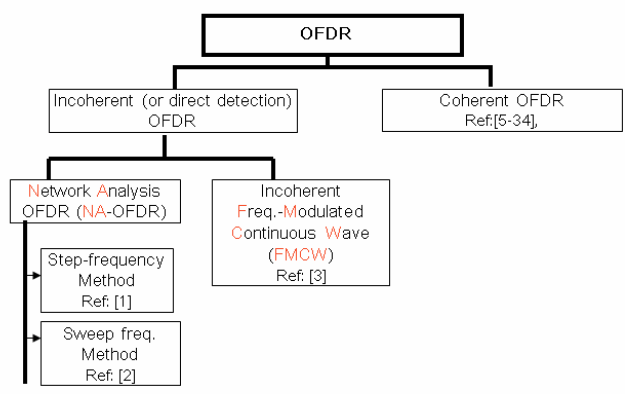

Figure 1. Classification of OFDR methods.

\subsection{Incoherent OFDR, I-OFDR}

In this method, a continuous wave (CW) optical carrier (probe signal) is intensity modulated by a constantamplitude RF signal whose frequency is changed periodically over a certain frequency range either stepwise (step-frequency method) [1] or continuously (sweep frequency method) [2]. This probe signal is then launched into DUT. Rayleigh backscattered and backreflected optical signals are detected as a function of modulation frequency and processed in a vector signal analyzer to obtain frequency response of the fiber. This is the reason why the group including step-frequency and sweep-frequency methods is globally called as Network Analysis OFDR (NA-OFDR). Fourier transform of the frequency response then gives the time-domain impulse response provided that the scanned frequency range is sufficiently large. Operation principles of NA-OFDR group are represented in Fig. 2a.

In an alternative sub-group called Incoherent Frequency-Modulated Continuous Wave (I-FMCW), the modulating RF signal is swept in frequency and the detected probe signal is mixed with the modulating RF signal in the electrical domain [3]. Resulting output that contains mixing products is then observed by means of an electrical spectrum analyzer. The frequency axis represents the delay times experienced by the probe signal. Knowing the speed of light within the fiber, time axis is converted into physical distance. Operation principles of I-FMCW are represented in Fig. 2b.
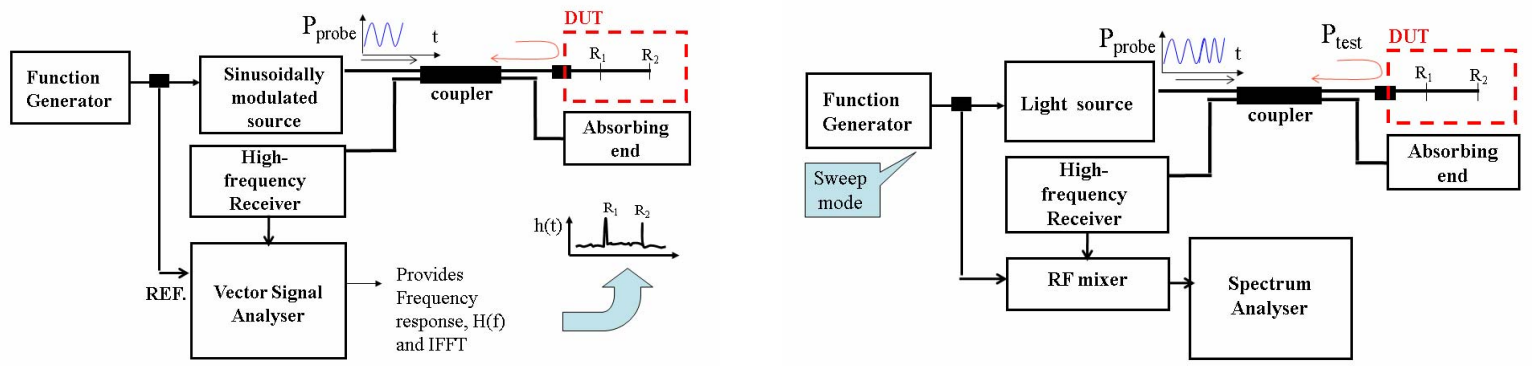

Figure 2. Operation principles of (a) NA-OFDR, (b) I-FMCW. 


\subsection{Coherent OFDR, C-OFDR}

In the C-OFDR category, the optical carrier frequency of the tunable laser source (TLS) is swept linearly in time without mode hops. Then, the frequency-modulated optical signal (probe signal) is split into two paths, one of which probes the DUT whereas the other is used as reference signal (or local oscillator). The reference signal returning from the reference mirror and test signal returning from the reflection sites in the test arm coherently interfere at the coupler. This interference signal contains the beat frequencies which appear as peaks at the network analyzer display after the Fourier transform of the time-sampled photocurrent.

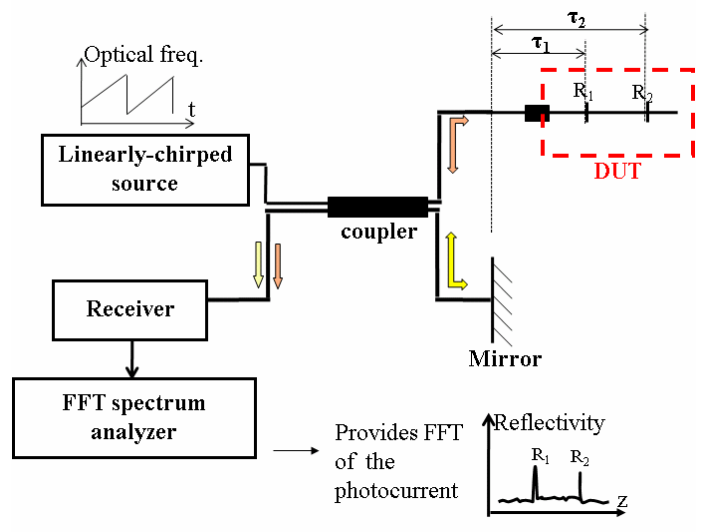

Figure 3. Operation principle of $C-O F D R$.

Using a linear optical frequency sweep, the measured beat frequencies can be mapped into a distance scale (the proportionality factor between beat frequency and the corresponding distance is determined by the rate of change of the optical frequency), while the squared magnitude of the signal at each beat frequency reveals the reflectivity of each reflection site [4]. This method is often called as coherent FMCW. Operation principle of $\mathrm{C}-\mathrm{OFDR}$ is represented in figure 3.

C-OFDR has got some advantages inherent to the coherent detection scheme [4]. First of all, the measured photocurrent is not proportional to the reflected optical power but to the square root of it, which permits the system to measure signals with large amplitude differences. Secondly, the receiver bandwidth (RF frequencies) is lower compared to the OTDR techniques reducing the noise level and increasing the dynamic range. Moreover, C-OFDR systems have got the ability to measure active devices (e.g. optical amplifiers) without saturation since only low power CW signals are used. Finally, no dead zone is observed in C-OFDR since the receiver does not saturate as in pulsed OTDR methods. However, C-OFDR has a problem in dealing with long measurement distances. The measurement distance is limited by the coherence length of the tunable light source.

\subsection{Applications of C-OFDR}

C-OFDR is useful for a vast range of different applications. Some typical application examples are listed below:

Characterization of optical components and modules: Although sub-millimeter resolution provided by the Optical Low Coherence Reflectometry (OLCR) technique is sufficient to analyse single, compact optical components, the measurement range of the OLCR is too short for more complicated optical modules including several pieces (e.g. WDMs, modulators,...). Thus, C-OFDR has to be employed with the case of such multicomponent modules and devices $[5,6]$.

Measurement of distributed gain in optical amplifiers: C-OFDR is a well-suited technique for the characterization of optical amplifiers for two reasons [7,8]. First of all, the amplified spontaneous emission (ASE) noise and the residual pump signal are filtered out thanks to the coherent detection scheme. Secondly, low power CW signal is used as test signal which does not saturate the optical amplifier-under-test.

Measurement of distributed birefringence: The polarization dependence of the coherent detection scheme can be exploited to measure birefringence [9] (or beat length [10]) in single-mode fibers and birefringence of polarization-maintaining fibers [11].

Biomedical imaging: There are big potentials for biomedical imaging applications by using high performance swept lasers. Imaging of human tissues by using about a $70 \mathrm{~nm}$ wavelength tuning range around $1300 \mathrm{~nm}$ [12], as well as an ophthalmic imaging system working in $1050 \mathrm{~nm}$ range [13] were recently reported.

\subsection{C-OFDR Solutions}

When realizing a C-OFDR set-up, the key component in the system is the narrow linewidth, mode-hop free tunable laser source (TLS) whose frequency can be chirped linearly in time without mode-hops over a large tuning range. Popularity and ease of application of C-OFDR techniques are expected to increase as the 
technology related to TLSs becomes more mature [4]. It is therefore of interest to give a complete list of optical sources proposed for the C-OFDR systems in the literature together with their achieved performances.

Tuned Nd:YAG ring laser [14]: For diagnostics of optical communication networks, a Nd:YAG laser operating at $1320 \mathrm{~nm}$ with a linewidth of $2 \mathrm{kHz}$ was used by Sorin et al. The optical frequency was chirped by ramping the temperature of the Nd:YAG crystal. Due to long coherence length of the laser, measurement ranges up to $50 \mathrm{~km}$ were realized. However, tuning range was limited in the experiment giving a spatial resolution of 380 meter.

Piezoelectrically tuned Nd:YAG ring laser [15]: Venkatesh et al. used in this option a piezoelectric rather than thermal tuning of a single-mode, Nd:YAG ring laser. The laser is tuned by piezoelectrically varying the gap between the two pieces making up the laser resonator. Fresnel reflections about $5 \mathrm{~cm}$ apart were resolved over a measurement range of $20 \mathrm{~m}$.

Current-directly-modulated distributed feedback laser diode (DFB-LD): This laser was claimed to be more reliable and less expensive than Nd:YAG lasers. A resolution of $50 \mathrm{~cm}$ was obtained for a measurement range $500 \mathrm{~m}$ by Shimizu et al by using a multiquantum well distributed feedback laser diode whose frequency is swept by injecting a control current [16].

Fiber grating external cavity laser [17]: A GaAs quantum well laser diode was spliced to a grating which is itself wrapped around a piezoelectric transducer cylinder to provide strain as a tuning mechanism. Any change in the cavity length induces a wavelength change. A resolution of $2 \mathrm{~m}$ over $115 \mathrm{~m}$ was obtained by Huang et al.

Three-section distributed feedback laser (DFB): In this option, Passy et al. [18] observed a resolution of $400 \mu \mathrm{m}$ for a measurement range of $10 \mathrm{~cm}$ by using a three-electrodes DFB laser at $1550 \mathrm{~nm}$ coupled to an external cavity. Optical frequency is swept by modulating the current of the central electrode with a triangular wavefunction. In a different arrangement realized by the same group, a double external cavity was coupled to the DFB laser. This provided a narrower linewidth $(200 \mathrm{kHz})$ which made possible to observe the Rayleigh backscattering over $400 \mathrm{~m}$ [19].

Externally phase-modulated Er-Yb laser [20]: Tsuji et al. demonstrated a resolution of $5 \mathrm{~m}$ over a range of $30 \mathrm{~km}$ incorporating an external electro-optic phase modulator to a Er-Yb laser oscillating at $1.55 \mu \mathrm{m}$.

Piezoelectrically tuned narrow linewidth fiber laser: In this option, the laser cavity is a $\mathrm{Yb}$-doped or Er/Yb codoped fiber spliced with a pair of fiber Bragg gratings at both extremities to form a laser resonator. The frequency of this fiber laser can be modulated through a small piezoelectric transducer, which expands or contracts the laser resonator in response to the applied voltage. The narrow linewidth of $10 \mathrm{kHz}$ provides longrange measurements. A resolution of $16 \mathrm{~cm}$ over a range of $150 \mathrm{~m}$ was demonstrated by Oberson et al [21]. Measurement of Rayleigh backscattering light over $95 \mathrm{~km}$ of fiber was demonstrated by Geng et al [22].

Mode-locked fiber laser [23]: The mode-locked fiber laser is in a unidirectional ring configuration constituting an erbium-doped fiber that is pumped by a laser diode $1480 \mathrm{~nm}$, an output coupler, isolators, a polarization controller, and a scanning Fiber Fabry-Pérot Filter (FPF). In this work, the FPF is modulated by a triangular waveform to produce a wavelength sweep over $20 \mathrm{~nm}$ centered at $1562 \mathrm{~nm}$. Spatial resolution of $100 \mu \mathrm{m}$ over a measurement range of several centimeters was demonstrated by $\mathrm{Oh}$ et al.

Frequency shifted feedback (FSF) laser [24]: Using FSF lasers, a resolution of $50 \mu \mathrm{m}$ over $1 \mathrm{~m}$ was reported by Ndiaye et al. for optical profilometry application.

\subsection{Challenges of C-OFDR}

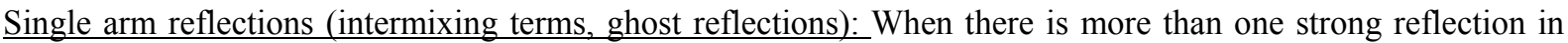
the test arm, some additional mixing terms between different reflectors are produced. These terms yield additional peaks on the beat spectrum making the interpretation difficult and do no correspond to real reflections. One way to eliminate this problem is to repeat the measurement with lower local oscillator signal by inserting attenuation into the reference arm. The intensity of the real reflections will decrease according to the attenuation, while the intermixing terms do not change [21]. Frequency shifters can also be used to have useful terms at shifted frequencies. Single arm reflections are not modified and stay at base band [25] and can be filtered out by a low-pass filter. Another practical way to remove intermixing terms is to add extra fiber delay into the test arm.

Polarization dependence: Because of the coherent detection scheme, the intensity of the measured signal is strongly affected by the state of polarisation (SOP) of the test light with respect to the reference light. Indeed, if the SOP of the reference and the test signals are orthogonal at the detector, the interference will be completely cancelled [26]. To avoid this dependence, the use of a polarisation diversity receiver was proposed [21]. In this method, the sum of the reference and test signal is split into two orthogonal polarization states, detected by two photodetectors. The intensities of both detected light signals are summed and result in a measured signal that is independent of the polarization. Another solution is to repeat a great number of measurements while changing the SOP of the reference arm and retaining the maximum signal intensity at the detector [27]. This technique requires less electronics and optical components than the above solution but is time consuming. 
Laser Intensity Noise: The power of reference signal is much greater than the power of test signal. In the case of single-photodetector systems, the reference signal produces a DC offset on the photocurrent on which are superposed all intensity fluctuations of the light source [18]. By using the well-known balanced-detection technique, laser intensity noise is eliminated. Balanced photodetectors consist of two well-matched photodiodes and a low noise transimpedance amplifier that generates an output voltage (RF signal) proportional to the difference between photocurrents of both photodiodes. Hence, common-mode noise that is present on both the reference and test signals is cancelled out and the RF output of the balanced detector provides directly the desired interference term.

Nonlinearity in the optical frequency modulation: When explaining operational principles of C-OFDR, we assumed that the optical frequency sweep is perfectly linear in time. However, the available lasers exhibit in practice fluctuations in their optical frequency tuning rate. Due to these nonlinear tuning characteristics, sampling of the interference signal with a constant spacing in time gives rise to a non-uniform sampling in optical frequency which, in turn, degrades the spatial resolution of the OFDR measurement. It is well known that this problem can be avoided by sampling the interference signal at equidistant instantaneous optical frequency points rather than equally spaced time intervals [28]. There are three approaches to realize this:

One entails the measurement of instantaneous optical frequency by using a so called auxiliary interferometer and corrects the nonlinearity on the laser source with some feedback mechanisms (e.g. adapting the modulating waveform according to the measured instantaneous optical frequency) [29-30]. The second method consists in realising the nonlinear frequency sweep suppression after data acquisition, using signal processing techniques [31]. As in the previous case, the instantaneous optical frequency is measured by an auxiliary interferometer, but contrary to the first method, this information is used for re-sampling of the interference signal with an accurate equidistant optical frequency grid after data acquisition. Recently reported Hilbert transform compensation method is a good example for the latter option [31]. In this method however, the need of phase-unwrapping used to obtain the phase of the detected light requires complex numerical algorithms. The last approach is most commonly implemented one and known as frequency sampling. As in the previous two approaches, the time varying optical frequency is simultaneously monitored by means of an auxiliary interferometer. The zero crossing points of the signal at the detector output of the auxiliary interferometer are used to generate trigger pulses which are then used as an external sampling clock on the test interferometer [32]. This approach has however a major drawback: the maximum DUT length is limited by the path difference of the auxiliary interferometer in order to satisfy Nyquist theorem [33].

Our group recently reported a new method for monitoring the nonlinear frequency sweep in high speed tunable laser sources [34]. The monitoring system comprises a Mach-Zehnder interferometer and simple signal processing steps. The time varying phase of the beat signal from a self-homodyne interferometer is first converted to an amplitude change. The envelope detection of this amplitude change directly gives the time varying optical frequency tuning rate. The tuning rate is then integrated to obtain time varying optical frequency. This system is able to measure optical swept-frequency in different kinds of tunable laser sources without any limitation in auxiliary interferometer length.

\section{CONCLUSIONS}

In this paper, we provide general background information on the optical frequency-domain reflectometry methods and their application areas. We also reviewed the existing C-OFDR solutions in terms of TLS used and performance characteristics achieved together with some solutions to the challenges observed in C-OFDR systems.

\section{ACKNOWLEDGEMENTS}

This research was supported by Fonds de la Recherche Scientifique-Crédit aux Chercheurs (FNRS).

\section{REFERENCES}

[1] J. Nakayama et al.: Optical fiber locator by the step frequency method, Appl. Opt., vol. 26, no. 3, pp. 440-443, 1987.

[2] D. Dolfi et al.: 5-mm-resolution OFDR using a coded phase-reversal modulator, Opt. Lett., vol. 13, no. 8, pp. 678-680, 1988.

[3] R. MacDonald, "Frequency domain optical reflectometer" Appl. Opt., vol. 20, no. 10, pp. 1840-1844,1981.

[4] D. Derickson, Fiber Optic Test and Measurement, Prentice Hall PTR, 1998.

[5] M. Wegmuller et al:: Overview of coherent reflectometry techniques: characterization of components and small systems, in Proc. Symposium on Optical Fiber Measurements, SOFM, Boulder, CO, 2000, pp. $155-160$.

[6] J. V. D. Weid et al.: Return loss measurements of WDM filters with tunable of coherent optical frequency domain reflectometry, Photonics Technol. Lett., vol. 9, no. 11, pp. 1508-1510, 1997. 
[7] M. Wegmuller et al.: Distributed gain measurements in Er-doped fibers with high resolution and accuracy using OFR, J. Lightwave Technol, vol. 18, no. 12, pp. 2127-2132, 2000

[8] J. Martins-Filho et al.: Dual-wavelength $(1050 \mathrm{~nm}+1550 \mathrm{~nm})$ pumped thulium doped fiber amplifier characterization by optical frequency-domain reflectometer, J. Lightwave Technol, vol. 18, no. 12, pp. 2127- 2132, 2000.

[9] J. Huttner et al.: Local birefringence measurements with optical frequency-domain reflectometry, Photonics Technol. Lett., vol. 10, no. 10, pp. 1458-1460, 1998.

[10] M. Wegmuller et al.: Distributed beat-length measurement in single-mode fibers with optical frequencydomain reflectometry, J. Lightwave Technol, vol. 20, no. 05, pp. 828-835, 2002.

[11] M. Froggatt et al.: Characterization of polarization-maintaining fiber using high-sensitivity optical frequency-domain reflectometer, J. Lightwave Technol, vol. 24, no. 11, pp. 4149-4154, 2006.

[12] S. Yun et al.: High-speed optical frequency-domain imaging, Opt. Express, vol. 11, no. 22, pp. 2953-2963, 2003.

[13] E. Lee et al.: In-vivo optical frequency-domain imaging of human retina and choroid, Opt. Express, vol. 14, no. 10, pp. 4403-4411, 2003.

[14] W. Sorin et al.: Coherent fmcw reflectometry using a temperature tuned Nd:YAG ring laser," Photonics Technol. Lett., vol. 2, no. 12, pp. 902-904, 1990.

[15] S. Venkatesh, W. Sorin, D. Donald, and H. B.L., "Coherent FMCW reflectometry using a piezoelectrically tuned Nd:YAG ring laser,” in Proc. OFS 8, Monterey, CA, 1991, p. Paper W.3.4.

[16] K. Shimizu et al.: Measurement of Rayleigh backscattering in single-mode fibers based on coherent OFDR employing DFB laser diode, Photonics Technol. Lett., vol. 3, no. 11, pp. 1039- 1041, 1991.

[17] K.-Y. Huang et al.: OFDR using a fiber grating external cavity laser, Photonics Technol. Lett., vol. 6, no. 12, pp. 1466-1468, 1994.

[18] R. Passy et al.: Experimental and theoretical investigations of coherent OFDR with semiconductor laser sources," J. Lightwave Technol, vol. 12, no. 9, pp. 1622-1630, 1994.

[19] J. von der Weid et al.: Mid-range coherent optical frequency domain reflectometry with a DFB laser diode coupled to an external cavity," J. Lightwave Technol, vol. 13, no. 5, pp. 954-960, 1995.

[20] K. Tsuji et al.: Coherent optical frequency domain reflectometry using phase-decorrelated reflected and reference lightwaves," J. Lightwave Technol, vol. 15, no. 7, pp. 1102-1109, 1997.

[21] P. Oberson et al.: Optical frequency domain reflectometry with a narrow linewidth fiber laser, Photonics Technol. Lett., vol. 12, no. 7, pp. 867-869, 2000.

[22] J. Geng et al.: Narrow linewidth fiber laser for 100-km optical frequency domain reflectometry, Photonics Technol. Lett., vol. 17, no. 9, pp. 1827-1829, 2005.

[23] M.S. Oh, H.S. Park, and B.Y. Kim: Optical frequency-domain reflectometry based on wavelength-swept mode-locked fiber laser," Photonics Technol. Lett., vol. 15, no. 2, pp. 266-268, 2003.

[24] C. Ndiaye, T. Hara, and H. Ito, "Profilometry using a frequency-shifted feedback laser," in Proc. Conference on Lasers and Electro-Optics (CLEO), Baltimore, Maryland, May 22 2005, Paper CThM2.

[25] J. Weid et al.: Self-heterodyne coherent optical frequency-domain reflectometer, Electronics Letters, vol. 31, no. 23, pp. 2037-2038, 1995.

[26] G. Mussi et al:: Polarization effects in coherent frequency-domain reflectometry, Photonics Technol. Lett., vol. 8, no. 11, pp. 1377-1384, 1996.

[27] J. Weid von der et al.: On the characterization of optical fiber network components with optical frequency domain reflectometry," J. Lightwave Technol, vol. 15, no. 7, pp. 1131-1141, 1997.

[28] U. Glombitza and E. Brinkmeyer, "Coherent frequency-domain reflectometry for characterization of single-mode integrated-optical waveguides,” J. Lightwave Technol, vol. 11, no. 8, pp. 1377-1384, 1993.

[29] L.-T. Wang et al.: Loss measurements in optical waveguide devices by coherent frequency modulated continuous-wave reflectometry, Optics Letters, vol. 18, no. 13, pp. 1095-1097, 1993.

[30] K. Iiyama, et al.: Linearizing optical frequency-sweep of laser diode for FMCW reflectometry, J. Lightwave Technol, vol. 14, no. 2, pp. 173-178, 1996.

[31] T.-J. Ahn and D. Kim, "Analysis of nonlinear frequency sweep in high-speed tunable laser sources using self-homodyne measurement and Hilbert transformation," Appl. Opt., vol. 46, no. 13, pp. 2394-2400, 2007.

[32] J. B. Soller et al.: High resolution optical frequency domain reflectometry for characterization of components and assemblies," Opt. Express, vol. 13, no. 2, pp. 666-674, 2005.

[33] E. Moore and R. McLeod, "Correction of sampling errors due to laser tuning rate fluctuations in sweptwavelength interferometry," Opt. Express, vol. 16, no. 17, pp. 13 139-13 149, 2008.

[34] K. Yuksel et al.: Analysis and suppression of nonlinear frequency modulation in an optical frequencydomain reflectometer, Opt. Express 17, 5845-5851 (2009). 